\title{
THE
}

\section{InAs(110)-p(1×1)-Sb(1 ML): Electronic Structure and Surface Bonding}

\author{
A. B. McLean \\ D. M. Swanston \\ D. N. Mcllroy \\ University of Rhode Island \\ David R. Heskett \\ University of Rhode Island, dheskett@uri.edu \\ R. Ludeke
}

See next page for additional authors

Follow this and additional works at: https://digitalcommons.uri.edu/phys_facpubs

Terms of Use

All rights reserved under copyright.

\section{Citation/Publisher Attribution}

McLean, A. B., Swanston, D. M., Mcllroy, D. N., Heskett, D., Ludeke, R., \& Munekata, H. (1995). InAs(110)$\mathrm{p}(1 \times 1)-\mathrm{Sb}(1 \mathrm{ML})$ : Electronic structure and surface bonding. Physical Review B, 51(20), 14271-14277. doi: 10.1103/PhysRevB.51.14271

Available at: http://dx.doi.org/10.1103/PhysRevB.51.14271

This Article is brought to you for free and open access by the Physics at DigitalCommons@URI. It has been accepted for inclusion in Physics Faculty Publications by an authorized administrator of DigitalCommons@URI. For more information, please contact digitalcommons-group@uri.edu. 


\section{Authors}

A. B. McLean, D. M. Swanston, D. N. Mcllroy, David R. Heskett, R. Ludeke, and H. Munekata

This article is available at DigitalCommons@URI: https://digitalcommons.uri.edu/phys_facpubs/242 


\title{
$\operatorname{InAs}(110)-p(1 \times 1)-\operatorname{Sb}(1 \mathrm{ML})$ : Electronic structure and surface bonding
}

\author{
A. B. McLean and D. M. Swanston \\ Department of Physics, Queen's University, Kingston, Ontario, Canada K7L 3N6 \\ D. N. McIlroy* and D. Heskett \\ Department of Physics, University of Rhode Island, Kingston, Rhode Island 02881 \\ R. Ludeke and H. Munekata \\ IBM Thomas J. Watson Research Center, P.O. Box 218, Yorktown Heights, New York 10598 \\ (Received 5 December 1994; revised manuscript received 13 February 1995)
}

\begin{abstract}
The electronic structure of the $\operatorname{InAs}(110)-p(1 \times 1)-\mathrm{Sb}(1 \mathrm{ML})$ system has been studied using angleresolved photoemission with a synchrotron light source. The InAs(110) surfaces were grown by molecular-beam epitaxy on GaAs(110) substrates. Four two-dimensional states were found and their dispersion along the $\overline{\Gamma X}$ and $\overline{\Gamma X^{\prime}}$ directions of the $1 \times 1$ surface Brillouin zone was determined. Although there is excellent overall agreement between the experimental energy bands and the predictions of a previously published tight-binding calculation, the bandwidth of two states, along the direction that is orthogonal to the $\mathrm{Sb}$ chains, is underestimated. A possible explanation for this is proposed.
\end{abstract}

\section{INTRODUCTION}

Since Skeath et al. discovered ${ }^{1,2}$ that $\mathrm{Sb}$ orders epitaxially on $\operatorname{GaAs}(110)$, the $\mathrm{GaAs}(110)-p(1 \times 1)-\mathrm{Sb}(1 \mathrm{ML})$ system has become one of the most intensively studied epitaxial overlayer systems. These studies have furthered our understanding of epitaxial adatom growth on GaAs(110) and of the nature of the surface chemical bond. Although there are several possible atomic geometries that provide the observed $1 \times 1$ overlayer symmetry, Skeath et al. identified two likely candidates that also satisfy electron-counting considerations. They proposed that $\mathrm{Sb}$ atoms form zigzag chains which are positioned either on top or between the Ga-As zigzag chains. The former structure is frequently called the $p^{3}$ geometry, because the valence electrons of every second. $\mathrm{Sb}$ atom within the overlayer chain form a $p^{3}$ hybrid which allows the atoms to bond to two other $\mathrm{Sb}$ atoms and a surface $\mathrm{Ga}$ atom. The latter structure puts the adsorbates close to the atomic positions that the bulk atoms would be in. Consequently, the model has become known as the continued-layer structure or the epitaxial continued-layer structure (ECLS). Soon after Skeath et al.'s experiments it was shown that the continuedlayer structure was favored by a dynamical analysis of low-energy electron-diffraction (LEED) intensities. ${ }^{3}$

Later, calculations of the surface electronic structure were reported. The calculations were performed using the pseudopotential ${ }^{4}$ and tight-binding methods. ${ }^{5-7}$ Furthermore, the dispersion of the surface states was measured by two groups using angle-resolved photoemission (ARPES). ${ }^{8-11}$

Tight-binding calculations ${ }^{6,7}$ identified a kind of bond at the surface which is not found in bulk III-V semiconductors, and which does not have a small molecule analog. It was argued ${ }^{6,7}$ that $\mathrm{Sb}$ intrachain bonding is provided by planar $\sigma$ bonds, formed from $\mathrm{Sb} p_{x}$ and $p_{y}$ orbit- als. The $\mathrm{Sb} p_{z}$ orbitals form a $\pi$-bonded manifold, and the manifold bonds diffusively with the substrate. (The coordinate system has $x$ and $y$ in the surface plane.) It is this diffuse bond that has no small molecule analog. Mailhiot, Duke, and Chadi ${ }^{6,7}$ ruled out the formation of $s p^{3}$ hybrids after considering the bond angles within the $\mathrm{Sb}$ chain. The $\mathrm{Sb}$ intrachain bond angle $\left(91^{\circ}\right)$ is close to the value that is produced by $p^{2}$ bonding $\left(90^{\circ}\right)$, and is much smaller than the tetrahedral bond angle $\left(109.47^{\circ}\right) .^{3}$ It was this fact that led Mailhiot, Duke, and Chadi ${ }^{6,7}$ to treat the surface bonding in terms of chain states, and this approach successfully reproduced the intrachain bond angle.

This description of the surface bonding has been contested by Manghi, Calandra, and Molinari, ${ }^{12}$ who argue that the most natural description of the surface bonding is in terms of $s p^{3}$ hybridization although the intrachain bond angle is smaller than expected. The $\mathrm{Sb}$ valence electrons form $s p^{3}$ hybrids which allow the atoms to bond to two $\mathrm{Sb}$ atoms within the chain, an atom in the substrate (cation or anion) leaving two electrons to form a lone-pair orbital. The bonding between the chain and substrate is provided by backbonds between the $\mathrm{Sb}$ atoms in the chain and the $\mathrm{Ga}$ and As atoms in the substrate. Manghi, Calandra, and Molinari ${ }^{12}$ argue that a $s p^{3}$ hybrid structure does not necessarily imply an intrachain bond angle of $109.47^{\circ}$, because the $\mathrm{Sb}$ atoms bond to nonequivalent atoms.

In principle, it should be possible to differentiate between these two bonding schemes using ARPES. For example, the dispersion of the state associated with the backbond should provide a quantitative measure of the chain-substrate coupling. If the chain-substrate bonding is provided by a conventional covalent backbond, the dispersion of this state perpendicular to the chain should be large. If the chain-substrate bonding is provided by diffuse coupling between the $\pi$ manifold and the $s p^{3}$ hy- 
brids, the state dispersion orthogonal to the chain should be smaller.

In this study we have examined the electronic structure of Sb monolayers on InAs(110). This system belongs to a larger family which includes $\mathrm{GaAs}(110) / \mathrm{Sb}, 8,10,11,13$ $\mathrm{GaP}(110) / \mathrm{Sb},{ }^{11}$ and $\operatorname{InP}(110) / \mathrm{Sb}^{14}$ Furthermore, although the surface bands have been calculated, ${ }^{7}$ to our knowledge the system has not been studied before with angle-resolved photoemission.

\section{EXPERIMENTAL DETAILS}

Experiments were performed at the U12B angleresolved photoemission beamline located at the National Synchrotron Light Source (Brookhaven National Laboratory, Upton, New York). ${ }^{15}$ The experimental chamber was equipped with a hemispherical electrostatic energy analyzer ${ }^{16}$ which has an angular acceptance of $\pm 2^{\circ}$. The combined energy resolution of the monochromator and electron analyzer was $\approx 150 \mathrm{meV}$.

The $n$-type, unintentionally doped, InAs(110) sample was grown by molecular-beam epitaxy (MBE) at IBM Yorktown Heights on a GaAs(110) substrate, and details of the crystal growth can be found elsewhere. ${ }^{17,18}$ Subsequently, clean, atomically ordered surfaces were produced by several sputter-anneal cycles. The sample was sputtered with a defocused beam of $1-\mathrm{kV}$ argon ions for $10 \mathrm{~min}$ and then annealed to $400^{\circ} \mathrm{C}$ for $5-10 \mathrm{~min}$. This process produced surfaces that displayed reproducible valence-band spectra, core-level spectra, and LEED patterns. Core-level photoemission spectra of the In $4 d$ core-level comprised only two spin-orbit split doublets (surface and bulk). There was no evidence, such as a low-binding-energy doublet in the In $4 d$ core level, to suggest that there were In clusters on the surface.

The $\mathrm{Sb}$ overlayers were evaporated from a welloutgassed boron-nitride effusion cell. During the evaporation the pressure did not exceed $3 \times 10^{-10}$ Torr, and the pressure during the experiment was $\approx 1 \times 10^{-10}$ Torr. The $\mathrm{Sb}$ coverage was calculated from timed exposures to the evaporant beam and correlated with the ML break point in the core-level intensity. After deposition, the overlayers were annealed at $250^{\circ} \mathrm{C}$ for $5 \mathrm{~min}$ to order the overlayer. After annealing, the overlayers displayed clear $1 \times 1$ low-energy electron-diffraction patterns. The samples were aligned so that the zigzag chains were vertical, perpendicular to the A vector of the synchrotron light.

\section{RESULTS}

In Fig. 1, the result of depositing $\mathrm{Sb}$ on $\operatorname{InAs}(110)$ is illustrated. The lower spectrum was collected from the clean InAs(110) surface. The analyzer was positioned to probe the $A_{5}$-anion-derived surface state at the $\bar{X}$ point of the $1 \times 1$ surface zone with $19.5-\mathrm{eV}$ light. The figure illustrates the effect of sequentially depositing $\mathrm{Sb}$ onto the clean surface. As the $\mathrm{Sb}$ coverage is increased, the emission in the vicinity of the $A_{5}$ state broadens and the centroid of the emission moves to lower binding energy (the small band-bending shift of $\approx 170 \mathrm{meV}$ has not been subtracted from the spectra). At coverages of $1 \mathrm{ML}$ the emission has broadened considerably. However, annealing the surfaces at $250^{\circ} \mathrm{C}$ for $5 \mathrm{~min}$ separates the emission located $\approx 1.75 \mathrm{eV}$ below $E_{F}$ into two well-defined states which have been labeled $S^{\mathrm{I}}$ and $S^{\mathrm{II}}$. The figure also reveals the presence of a very intense feature $S^{\mathrm{III}} \approx 3 \mathrm{eV}$ below $E_{F}$ and a shoulder $S^{\mathrm{IV}}$ at $\approx 3.8 \mathrm{eV}$ below $E_{F}$. Although it is unusual for a valence-band spectrum to be entirely dominated by surface-state emission, we will show later that, at the $\bar{X}$ point, three of the four states $\left(S^{\mathrm{I}}, S^{\mathrm{III}}\right.$, and $\left.S^{\mathrm{IV}}\right)$ lie within gaps in the projected bulk band structure of InAs. This provides strong evidence that they are surface states. In contrast, $S^{\text {II }}$ appears to be in resonance with the bulk continuum at this point.

Three of the four states mentioned above $\left(S^{\mathrm{I}}, S^{\mathrm{II}}\right.$, and $S^{\mathrm{IV}}$ ) appear to disperse symmetrically about $\bar{X}$, although it was not possible to follow the states deep within the bulk continuum. At first inspection the dispersion of the $S^{\text {III }}$ state about $\bar{X}$ appears to lack the requisite mirror symmetry (see below). However, we believe that, away from the $\bar{X}$ point, the $S^{\mathrm{III}}$ state overlaps a bulk transition, if photon energies in the range $19.5-22.0 \mathrm{eV}$ are used to

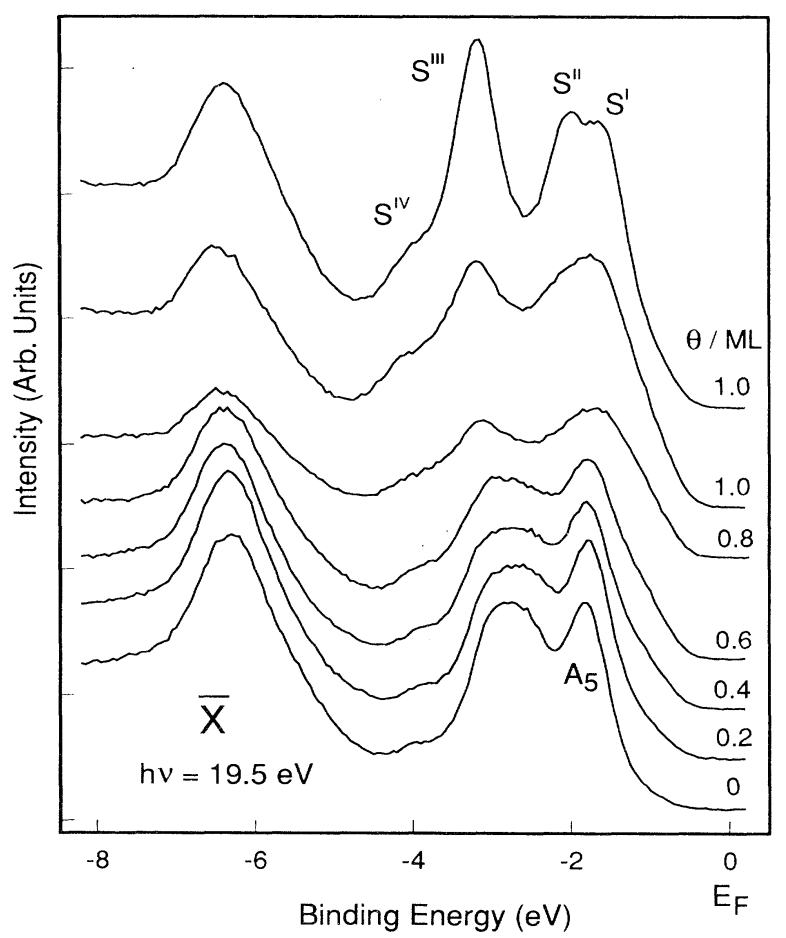

FIG. 1. Photoemission spectra from the clean InAs(110) surface (bottom curve) and for the same surface with various $\mathrm{Sb}$ coverages which are indicated on the figure in ML. With the exception of the top curve, the Sb overlayers were not annealed after deposition. The top spectrum is a ML coverage which was annealed at $250^{\circ} \mathrm{C}$ for $5 \mathrm{~min}$ to order the overlayer. The intensity of the state located at $\approx 3 \mathrm{eV}$ below $E_{F}$ increases, and the broad feature located $\approx 1.75 \mathrm{eV}$ below $E_{F}$ splits into two welldefined states $\left(S^{\mathrm{I}}\right.$ and $\left.S^{\mathrm{II}}\right)$. Annealing the surface also reduces the diffuse background in the LEED pattern. 
probe the state. This fact makes it difficult to determine the binding energy of $S^{\text {III }}$ with a high degree of precision.

In Fig. 2 the emission from the clean InAs(110) surface is shown in the lower portion of the figure. The analyzer is positioned to probe the intense feature located $2 \mathrm{eV}$ below $E_{F}$ at the $\bar{X}^{\prime}$ point with $19.5-\mathrm{eV}$ light. Directly above this is a spectrum from the annealed ML system which was taken with the same photon energy and with the analyzer in the same position. Although there is a remnant of the intense feature that is present on the lower spectrum, there is additional emission to both lower and higher binding energies. These additional new features have been labeled $S^{\mathrm{I}}$ and $S^{\mathrm{IV}}$, respectively. From studies at other photon energies and from comparison with spectra taken from the clean surface, ${ }^{19}$ we know that the emission between these two states arises from a bulk feature and at least one other two-dimensional, surface-localized state $\left(S^{\mathrm{II}}\right)$ which is located $\approx 500 \mathrm{meV}$ below $S^{\mathrm{I}}$ at $\bar{X}^{\prime}$. We found little evidence for an additional surface band (e.g., $S^{\mathrm{III}}$ ) located between $S^{\mathrm{II}}$ and $S^{\mathrm{IV}}$. (The labeling of the surface features will be explained below.) The upper three spectra in Fig. 2 were obtained by moving the analyzer further off-normal, so that the region of reciprocal space beyond $\bar{X}^{\prime}$ toward the $\bar{\Gamma}$ point of

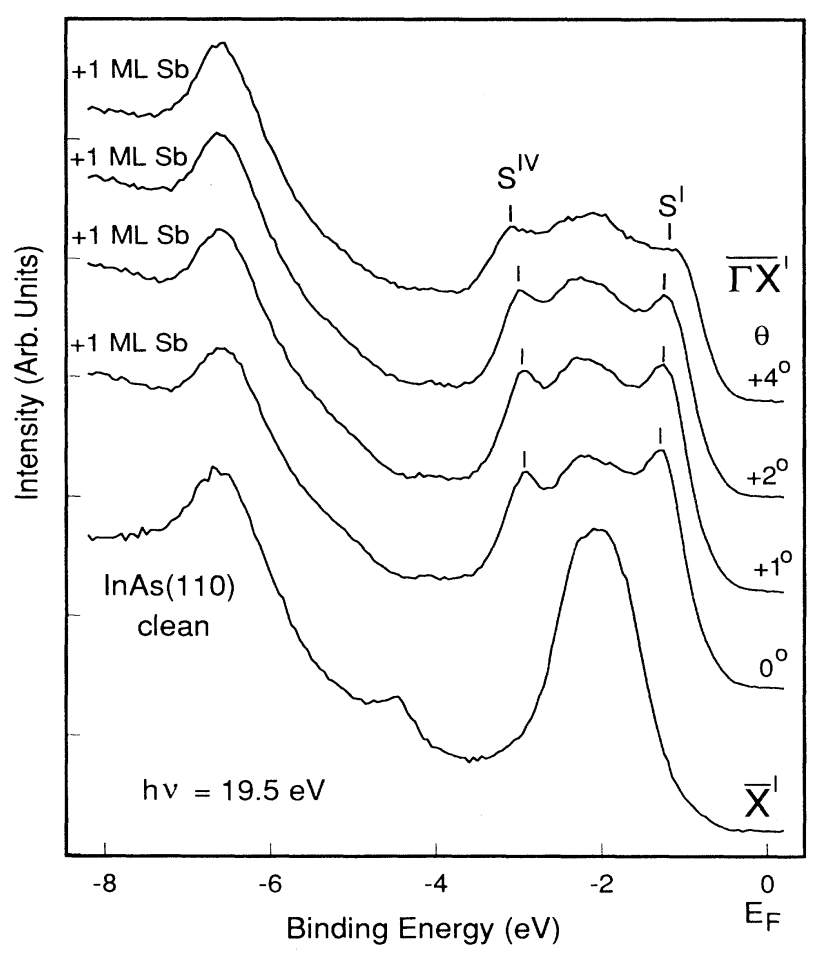

FIG. 2. Photoemission spectra from the clean InAs(110) surface (bottom curve) and from the $\operatorname{InAs}(110)-p(1 \times 1)-\mathrm{Sb}(1 \mathrm{ML})$ system (all other curves). In the bottom curve the analyzer is positioned to probe the large peak $2 \mathrm{eV}$ below $E_{F}$ at the $\overline{X^{\prime}}$ point. In the other spectra the analyzer is moved beyond $\overline{X^{\prime}}$, by the angle indicated on each spectrum, toward the $\bar{\Gamma}$ point of the second zone. the second zone is probed. As the analyzer is moved beyond $\bar{X}^{\prime}, S^{\mathbf{I}}$ disperses upwards and $S^{\mathrm{IV}}$ disperses downwards.

The second state $\left(S^{\mathrm{II}}\right)$ is visible in Fig. 3. All the spectra were collected with $19.5-\mathrm{eV}$ light, and the analyzer was positioned along the $\overline{\Gamma X}$ direction. Although three states are visible with this photon energy, the initial-state dispersion along $\overline{\Gamma X}$ is very shallow. Both $S^{\mathrm{I}}$ and $S^{\mathrm{II}}$ are clearly visible near the $\bar{X}$ point $\left(\approx 23^{\circ}\right)$. Of the three, $S^{\mathrm{IV}}$ is the only state visible near $\bar{\Gamma}$.

In Fig. 4 the strong dispersion of the $S^{\mathrm{IV}}$ state along the $\overline{\Gamma X^{\prime}}$ direction is clearly visible. The $S^{\mathrm{IV}}$ state reaches the $X^{\bar{\top}}$ point at $\theta \approx 15^{\circ}$. The $S^{\mathrm{I}}$ state is also visible as a shoulder near this $X^{\top}$ point with the photon energy.

The two-dimensional nature of the three states $S^{\mathrm{I}}, S^{\mathrm{II}}$, and $S^{\mathrm{IV}}$, is illustrated in Fig. 5. The upper spectrum was collected with $19.5-\mathrm{eV}$ light. The analyzer was positioned $16^{\circ}$ off-normal along $\overline{\Gamma X^{\prime}}$. The lower spectrum was collected with $22-\mathrm{eV}$ light with the analyzer $14.7^{\circ}$ offnormal. Both energy distribution curves can be modeled using four Voigt functions ${ }^{20}$ and a function to describe

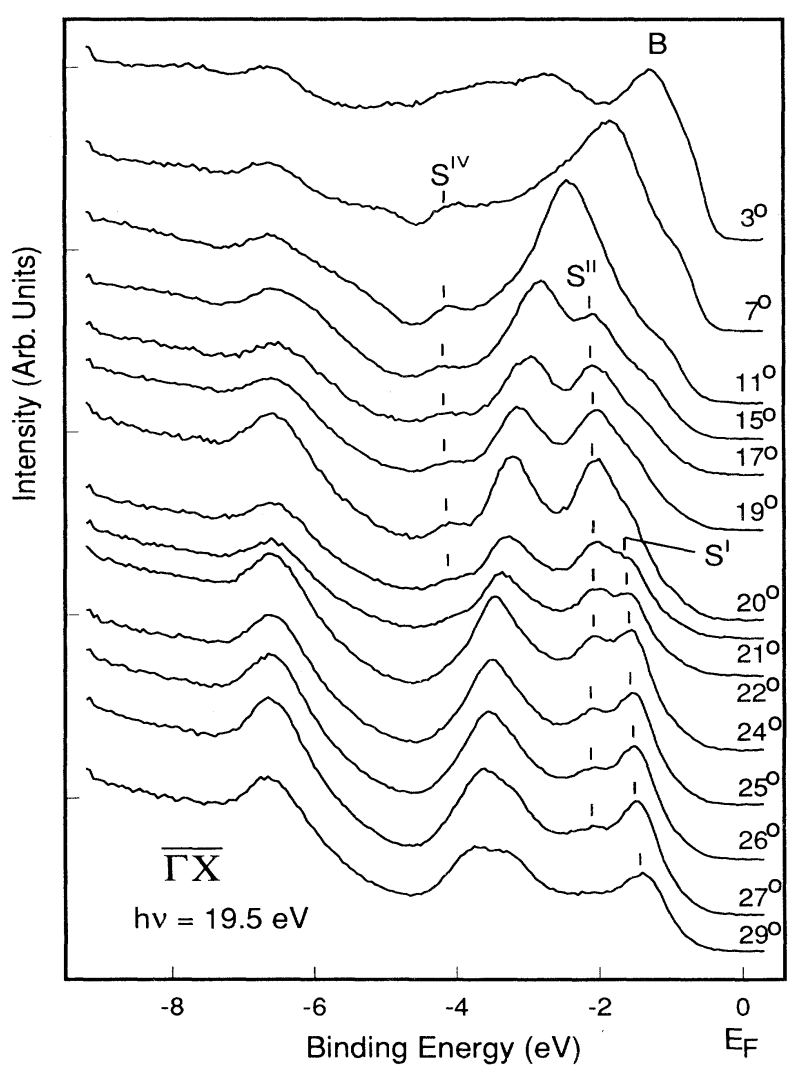

FIG. 3. Photoemission spectra from the $\operatorname{InAs}(110)-p(1 \times 1)-$ $\mathrm{Sb}(1 \mathrm{ML})$ system taken with 19.5 -eV light along $\overline{\Gamma X}$. In this energy range three two-dimensional, surface-localized states are visible, although their dispersion is very shallow. Near the zone center the emission from both $S^{\mathrm{I}}$ and $S^{\mathrm{II}}$ is swamped by bulk emission (marked $B$ ). The numbers on the curves indicate the position of the analyzer relative to the surface normal. 
the secondary electron background. ${ }^{21}$ The four Voigt functions are associated with the three two-dimensional states that are described above and a bulk state (marked $B$ in the figure). Nonlinear least-squares analysis allows the position of the states to be established when the peaks are not completely resolved in the energy distribution curve. The three states $S^{\mathrm{I}}, S^{\mathrm{II}}$, and $S^{\mathrm{IV}}$ are probed at values of $k_{\|}$which lie in the range $0.63 \pm 0.05,0.62$ \pm 0.05 , and $0.61 \pm 0.04 \AA^{-1}$, respectively. The bindingenergy shifts for the surface states are in all cases less than the experimental error (i.e., $30 \mathrm{meV}$ ). This confirms their two-dimensional nature. In contrast, the state that we have labeled $B$ in Fig. 5 shifts by $\approx 100 \mathrm{meV}$, illustrating that it is truly a bulk state. As we shall argue below, by comparing our results with tight-binding calculations, it is possible to make a one-to-one assignment of the experimental and theoretical surface bands. This leads us to believe that there may be another surface band between the states we have labeled $S^{\text {II }}$ and $S^{\text {IV }}$. However, we were not able to locate this band because core-level emission from second-order light limited the range of photon energies we could use in the experiment.

The results of our band-mapping studies are presented in Fig. 6. The dispersion of the three states (dots) is plot-

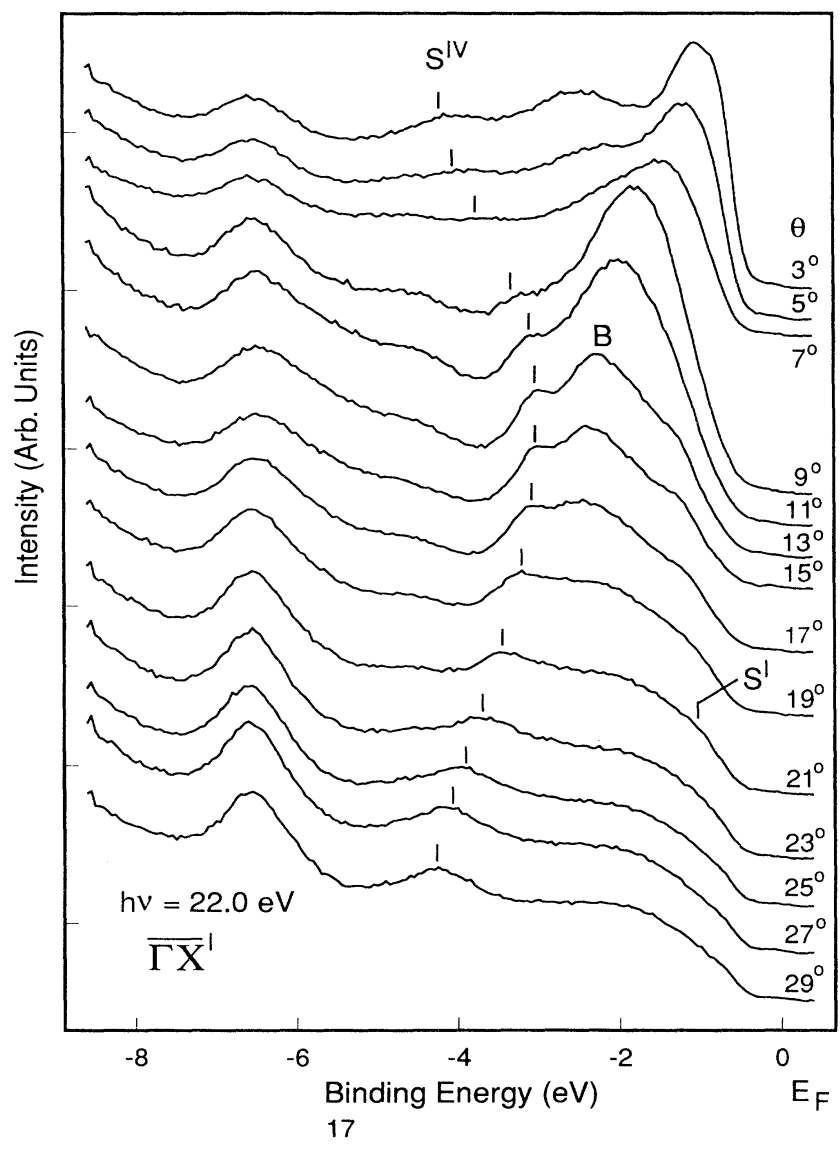

FIG. 4. The dispersion of the $S^{\mathrm{IV}}$ state is clearly visible along the $\overline{\Gamma X^{\prime}}$ direction. The $S^{\text {IV }}$ state hits the $\overline{X^{\prime}}$ point $\approx 16^{\circ}$ offnormal.

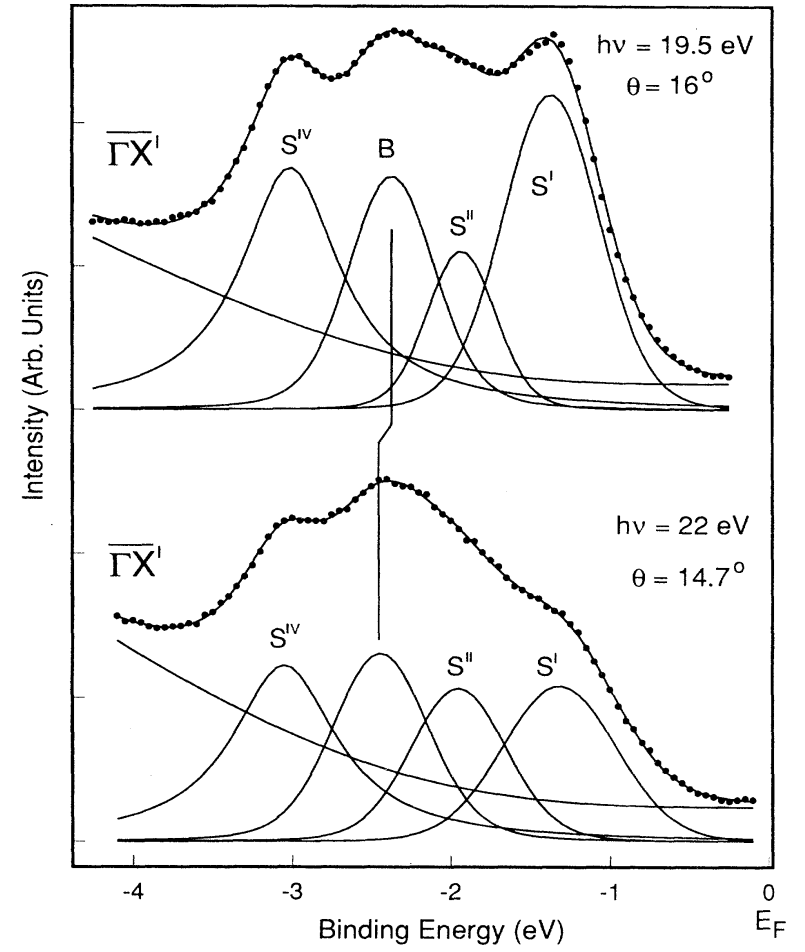

FIG. 5. The two-dimensional nature of the three states $S^{\mathrm{I}}$, $S^{\mathrm{II}}$, and $S^{\mathrm{IV}}$ is illustrated. The analyzer was positioned so that the projection of the free-electron momentum vector onto the surface plane was approximately the same in both cases. The states labeled $S^{\mathrm{I}}, S^{\mathrm{II}}$, and $S^{\mathrm{IV}}$ (within the experimental uncertainty) do not change binding energy, whereas the state labeled $B$ does. This indicates that the feature labeled $B$ arises from a band which is truly three dimensional.

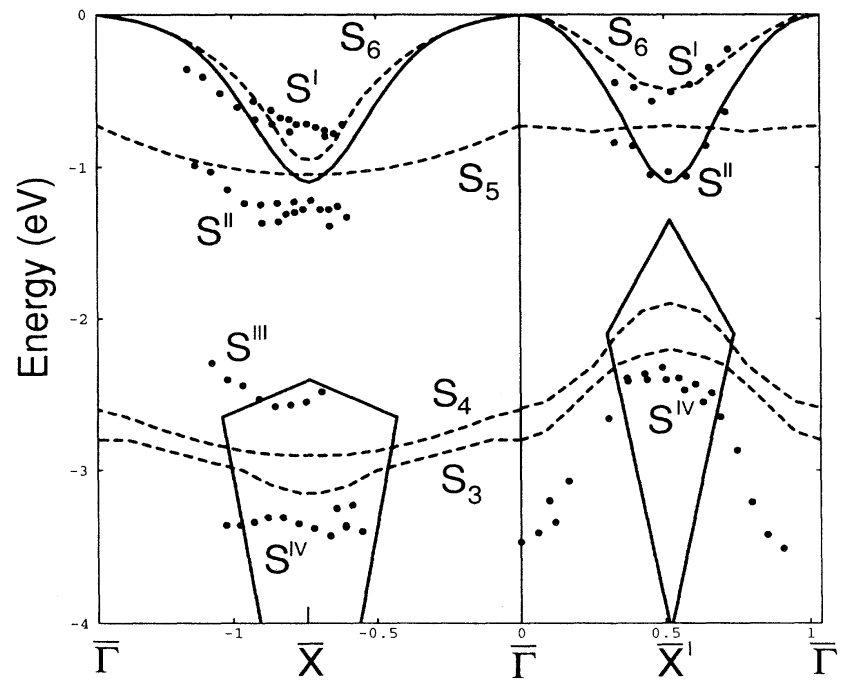

FIG. 6. The results of band-mapping studies along the $\overline{\Gamma X \Gamma}$ and $\overline{\Gamma X^{\prime} \Gamma}$ directions. The points are the experimental initialstate dispersions, which were extracted from energy distribution curves collected with 19.5- and 22.0-eV light. The dashed lines are the calculated surface eigenvalue spectrum of the $S_{3}-S_{6}$ states (Ref. 7). The full lines are the edge of the projected bulk bands (Ref. 7). The energy scale is referenced to the valenceband maximum. For clarity the projected bulk bands have not been shaded. 
ted along the $\overline{\Gamma X^{\prime}}$ and $\overline{\Gamma X}$ high-symmetry directions, and compared with the results of a tight-binding calculation. ${ }^{7}$ The experimental points were extracted from energy distribution curves that were collected at both 19.5 and 22.0 $\mathrm{eV}$. The dashed lines are the calculated surface bands (labeled $S_{3}-S_{6}$ ), and the full lines are the edge of the projected bulk bands.

Several points are worthy of note. (1) Around $\overline{X^{\prime}}$ the dispersion and binding energy of $S^{\mathrm{I}}$ are in excellent agreement with the calculated dispersion of the $S_{6}$ band. (2) At $\bar{X}$ the $S^{\mathrm{I}}$ state is within $250 \mathrm{meV}$ of $S_{6}$, and the dispersion of the $S^{\mathbb{I}}$ band is shallower than the dispersion of the $S_{6}$ band. (3) The dispersion of $S^{\mathrm{II}}$ appears to follow the projected bulk band edge in the vicinity of $\overline{X^{\prime}}$ point, and there seems to be little correspondence with the calculated dispersion of the $S_{5}$ band in this region of the surface zone. (4) At the $\bar{X}$ point $S^{\mathrm{II}}$ is at higher binding energy than $S_{5}$, but the dispersion of the band along $\overline{\Gamma X}$ is in excellent agreement with the dispersion of the $S_{5}$ band. (5) The $S^{\mathrm{III}}$ state appears within a projected bulk band gap at the $\bar{X}$ point of the surface zone. Although the dispersion of this state about $\bar{X}$ does not appear to be completely symmetric, we believe this is because the state overlaps a bulk transition in the narrow range of photon energies that we used in the experiment. This makes it particularly difficult to extract the binding energy of the state. (6) At $\overline{X^{\prime}}$, the binding energy of the $S^{\mathrm{IV}}$ band is within $200 \mathrm{meV}$ of $S_{3}$. Consequently, the experimental state has been labeled $S^{\mathrm{IV}}$. (7) The dispersion of $S^{I V}$ along $\overline{\Gamma X^{\prime} \Gamma}$ is much larger than the dispersion of either of the $S_{3}$ or $S_{4}$ bands. (8) The binding energy of $S^{\mathrm{IV}}$ at $\bar{X}$ is within $250 \mathrm{meV}$ of $S_{3}$.

\section{DISCUSSION}

It is obvious from Fig. 6 that there is a good level of agreement between the experimental and calculated bands. $^{7}$ This allows a fairly straightforward assignment of the experimental bands to be made (e.g., $S^{\mathrm{I}}=S_{6}$, $S^{\mathrm{II}}=S_{5}, S^{\mathrm{III}}=S_{4}$, and $S^{\mathrm{IV}}=S_{3}$ ). In the following discussion it must be borne in mind that the tight-binding bands that we have reproduced in Fig. 6 were calculated a decade ago, long before the dispersion of the bands were experimentally determined. Furthermore the tightbinding calculation represents one of the first attempts to predict the surface-state eigenvalue spectrum. Considering both these facts the agreement between experiment and theory is very impressive. Although there are some differences in detail, that will be discussed at length below, the predictions of the calculation regarding the number and location of the bands are in good overall agreement with experiment.

We will now turn to a detailed comparison between experiment and theory. First, we notice that the binding energy and position of the $S^{\mathrm{I}}$ state matches the predicted binding energy and dispersion of the $S_{6}$ state. This is particularly true along the $\overline{\Gamma X^{\prime}}$ azimuth. Along $\overline{\Gamma X}$ the calculated dispersion appears to be overestimated, although the location of the state is in good agreement with experiment.

Second, although the calculations reproduce the dispersion of the $S^{\text {II }}$ state remarkably well about the $\bar{X}$ point, the calculated binding energy appears to be approximately $250 \mathrm{meV}$ too small. About the $\overline{X^{\prime}}$ point the situation is quite different. The calculation predicts that the dispersion of $S_{5}$ should almost be flat, whereas the dispersion of $S^{\mathrm{II}}$ is very pronounced and it appears to follow the projected bulk band edge. Although it is not uncommon to see indirect emission from band edges in angle-resolved photoemission spectra (e.g., Ref. 22), we have no reason to attach any special significance to this.

Third, the lower bands $S_{3}$ and $S_{4}$ are assigned to the experimental $S^{\mathrm{IV}}$ and $S^{\mathrm{III}}$ bands. We note that the binding energy and the dispersion of the $S^{\text {II }}$ band is in very good agreement with the binding energy and dispersion of the $S_{3}$ band about the $\bar{X}$ point. However, the splitting between the $S^{\mathrm{III}}$ and the $S^{\mathrm{IV}}$ bands at $\bar{X}$ is much greater than the corresponding splitting of the $S_{3}$ and $S_{4}$ bands. As we have mentioned above, in the experimental energy distribution curves the $S^{\mathrm{III}}$ state overlaps a bulk transition. Consequently, the location of the $S^{\text {III }}$ state about $\bar{X}$ is only known to within $\approx 100 \mathrm{meV}$. We believe that the close proximity of the bulk transition produces the asymmetry of the measured initial-state dispersion about the $\bar{X}$ point. Both $S^{\mathrm{III}}$ and $S^{\mathrm{IV}}$ disperse into projected gaps in the bulk band structure. Consequently they must be surface-related features.

Fourth, the curvature of the $S^{\mathrm{IV}}$ band about $\overline{X^{\prime}}$ is in excellent agreement with the calculations, and the predicted binding energy of this band is also in good agreement with experiment. However, the bandwidth of the $S^{\mathrm{IV}}$ long $\overline{\Gamma X^{\prime}}$ is almost a factor of 2 larger than the bandwidth of either $S_{3}$ or $S_{4}$.

Perhaps one of the most striking differences between the measured and calculated surface-state dispersions presented in Fig. 6 is the difference between the predicted and measured bandwidths along the $\overline{\Gamma X^{\prime}}$ direction. The experimental dispersion of both the $S^{\mathrm{II}}$ and $S^{\mathrm{IV}}$ states are substantially larger than the calculated bandwidths of the $S_{3}, S_{4}$, and $S_{5}$ bands. Consequently, the tight-binding calculation appears to underestimate the strength of the wave-function overlap in the direction orthogonal to the $\mathrm{Sb}$ chains. In the direction that is parallel to the $\mathrm{Sb}$ chains, the initial-state dispersion of the surface bands is reproduced remarkably well by the calculation.

The dispersion of the surface bands have also been measured in the $\operatorname{InP}(110)-p(1 \times 1)-\mathrm{Sb}(1 \mathrm{ML})$ system, $^{14}$ and the same trend is found. Although there is good overall agreement between the tight-binding calculation ${ }^{7}$ and experiment, the calculations underestimate the dispersion of the low-lying states along $\overline{\Gamma X^{\prime}}$ (see Table I).

We note that although Mårtensson et al. ${ }^{8}$ performed a very thorough mapping of the GaAs(110)-p $(1 \times 1)-\mathrm{Sb}(1$ ML) system, they were unable to measure (presumably because of overlap with bulk states) the bandwidth of the lower-lying surface states along $\overline{\Gamma X^{\prime}}$. Consequently, at this time, it is not possible to determine whether this is a general trend. However, in the following we will briefly explore the implications of this finding.

As we emphasized above, the experimental picture is far from complete. However, the first band-mapping studies of the III-V (110)- $p(1 \times 1)$-Sb (1 ML ) family sug- 
TABLE I. A comparison between the experimentally determined bandwidth of the $S^{\text {III }}$ state with the calculated dispersion (Ref. 7) of the $S_{3}$ and $S_{4}$ bands along $\overline{\Gamma X^{\prime}}$. All bandwidths are measured in $\mathrm{eV}$. In Ref. 14, McGovern et al. studied the $\operatorname{InP}(110)-p(1 \times 1)-\mathrm{Sb}(1 \mathrm{ML})$ system and identified a state which was labeled $\delta$. It was shown to have surface provenance, and it clearly corresponds to the $S^{\mathrm{IV}}$ state described in the main text.

\begin{tabular}{cccc}
\hline \hline System & Experimental & $S_{3}$ & $S_{4}$ \\
\hline $\operatorname{InP}(110) / \mathrm{Sb}$ & 1.63 (Ref. 14) & 0.53 & 0.53 \\
$\operatorname{InAs}(110) / \mathrm{Sb}$ & 1.20 (this study) & 0.68 & 0.73 \\
\hline \hline
\end{tabular}

gest that although the tight-binding calculations performed by Mailhiot et al. 6,7 are in good overall agreement with experiment, they do underestimate the dispersion of the lower-lying surface states along $\overline{\Gamma X^{\prime}}$. In the direct lattice, this direction is orthogonal to the $\mathrm{Sb}$ chains and our results suggest that the wave-function overlap along this direction is significantly larger than previously thought. Although the nearest-neighbor tight-binding method should reproduce the strength of the chainsubstrate interaction, it is possible that it may underestimate the strength of the chain-chain interaction which may be mediated by subsurface valence electrons.

\section{CONCLUSIONS}

The surface-state eigenvalue spectrum of the InAs(110)-( $1 \times 1)-S b(1 \mathrm{ML})$ system has been studied using
ARPES and compared with the results of some tightbinding calculations. ${ }^{6,7}$ Four surface bands $\left(S^{\mathrm{I}}, S^{\mathrm{II}}, S^{\mathrm{III}}\right.$, and $S^{\mathrm{IV}}$ ) were detected and their dispersion along the $\overline{\Gamma X^{\prime}}$ and $\overline{\Gamma X}$ directions of the surface Brillouin zone was measured. We found very good overall agreement between the experimental and the calculated bands., ${ }^{6,7}$ Considering that the surface bands were calculated a decade before band-mapping studies, such as this one, could provide information about the surface-state eigenvalue spectrum, the agreement between the tight-binding calculations and experiment is very impressive. A detailed comparison between the experimental and calculated bands revealed that the bandwidth of two of the lower-lying experimental bands along the $\overline{\Gamma X^{\prime}}$ direction is larger than the tight-binding calculation predicted. One possible explanation for this discrepancy was proposed.

\section{ACKNOWLEDGMENTS}

This research was supported by the Natural Sciences and Engineering Research Council of Canada. The National Synchrotron Light Source, located at Brookhaven National Laboratory is sponsored by the U. S. Department of Energy (Division of Materials Sciences and Chemical Sciences). We would like to thank P. Bruhwiler for expert experimental assistance and C. B. Duke for his critical reading of the original manuscript.
*Present address: Behlen Laboratory of Physics, Center for Materials Research and Analysis, University of Nebraska-Lincoln, Lincoln, Nebraska 68588-0111.

${ }^{1}$ P. Skeath, C. Y. Su, I. Lindau, and W. E. Spicer, J. Vac. Sci. Technol. 17, 874 (1980).

${ }^{2}$ P. Skeath, I. Lindau, C. Y. Su, and W. E. Spicer, J. Vac. Sci. Technol. 19, 556 (1981).

${ }^{3}$ C. B. Duke, A. Paton, W. K. Ford, A. Kahn, and J. Carelli, Phys. Rev. B 26, 803 (1982).

${ }^{4}$ C. M. Bertoni, C. Calandra, F. Manghi, and E. Molinari, Phys. Rev. B 27, 1251 (1983).

${ }^{5}$ P. Skeath, C. Y. Su, W. A. Harrison, I. Lindau, and W. E. Spicer, Phys. Rev. B 27, 6246 (1983).

${ }^{6}$ C. Mailhiot, C. B. Duke, and D. J. Chadi, Phys. Rev. Lett. 53, 2114 (1984).

${ }^{7}$ C. Mailhiot, C. B. Duke, and D. J. Chadi, Phys. Rev. B 31, 2213 (1985).

${ }^{8}$ P. Mårtensson, G. V. Hansson, M. Lähdeniemi, K. O. Magnusson, S. Wiklund, and J. M. Nicholls, Phys. Rev. B 33, 7399 (1986).
${ }^{9}$ A. Tulke and H. Lüth, Surf. Sci. 178, 131 (1986).

${ }^{10}$ M. Mattern-Klosson, R. Strümpler, and H. Lüth, Phys. Rev. B 33, 2559 (1986)

${ }^{11}$ A. Tulke, M. Mattern-Klosson, and H. Lüth, Solid State Commun. 59, 303 (1986).

${ }^{12}$ F. Manghi, C. Calandra, and E. Molinari, Surf. Sci. 184, 449 (1987).

${ }^{13}$ A. Tulke, M. Mattern-Klosson, and H. Lüth, Solid State Commun. 59, 303 (1986).

${ }^{14}$ I. T. McGovern, R. Whittle, D. R. T. Zahn, C. Müller, C. Nowak, A. Cafolla, and W. Braun, J. Phys. Condens. Matter 3, S367 (1991).

${ }^{15}$ B. P. Tonner, Nucl. Instrum. Methods 172, 133 (1980).

${ }^{16}$ C. L. Allyn, T. Gustafsson, and E. W. Plummer, Rev. Sci. Instrum. 49, 1197 (1978).

${ }^{17}$ H. Munekata, L. L. Chang, S. C. Woronick, and Y. H. Cao, J. Cryst. Growth 81, 237 (1987).

${ }^{18}$ H. Munekata, A. Segmüller, and L. L. Chang, Appl. Phys. Lett. 51, 587 (1987).

${ }^{19}$ D. M. Swanston, A. B. McLean, D. N. McIlroy, D. Heskett, 
R. Ludeke, H. Munekata, M. Prietsch, and N. J. DiNardo, Surf. Sci. 312, 361 (1994).

${ }^{20}$ A. B. McLean, C. E. J. Mitchell, and D. M. Swanston, J. Electron Spectrosc. Relat. Phenom. 69, 125 (1994).
${ }^{21}$ S. Tougaard, Surf. Sci. 216, 343 (1989).

${ }^{22}$ K. O. Magnusson, S. A. Flodström, and P. E. S. Persson, Phys. Rev. B 38, 5384 (1988). 